\title{
Modification of Optical Interferometers with Functional Polymers and Hydrogels for Sensing of Protein Biomarkers
}

\author{
Steffen Kurzhals $^{1}$, Eva Melnik ${ }^{1}$, Florian Strasser ${ }^{1}$, Paul Muellner ${ }^{1}$, Guenther Koppitsch², \\ Rudolf Heer ${ }^{1}$, Rainer Hainberger ${ }^{1}$ \\ ${ }^{1}$ AlT Austrian Institute of Technology $\mathrm{GmbH}$, Giefinggasse 4, 1210 Vienna, Austria \\ 2 ams AG, Tobelbader Straße 30, 8141 Premstätten, Austria \\ steffen.kurzhals@ait.ac.at
}

\begin{abstract}
:
Surface modification of Mach-Zehnder interferometers with biotin-modified polyethylenimine (PEI-B) and PEI-B loaded hydrogels can give access to biosensors combining highly binding matrices with low non-specific interactions, useful for protein biosensing. Permeability of hydrogel layers prepared by photocrosslinking depends on used precursors as well as crosslinking conditions. Protein loading capacity is probed for both functionalization strategies showing high initial response for directly modified surfaces and linear response with receptor concentration for sensors modified with receptor loaded hydrogels. Further signal increase can be achieved by combining receptor modified surfaces with receptor loaded hydrogel layers.
\end{abstract}

Key words: Optical waveguide, Mach-Zehnder Interferometer, Interferometry, Hydrogel, Protein Biosensing.

Surface modification of photonic waveguides with functional polymers and hydrogels enables to obtain biosensors that combine highly binding matrices with low non-specific interactions, useful for the detection of protein biomarkers.

For probing the protein binding capacity of such photonic biosensors we apply as receptor biotin-modified polyethylenimine (PEI-B) that is characterized by its highly specific biotinstreptavidin interaction. Two different functionalization strategies are investigated, a) binding PEI-B molecules directly to the surface and $b$ ) incorporating the receptor in a surfaceattached, dextran-based hydrogel film. Direct surface modification ensures high receptor density. The application of hydrogel films has the advantage that they can serve both as inert binding matrix and semipermeable separation layer.

Hydrogel precursor solutions with different viscosities and photo cross-linker fraction were prepared by etherification of dextran. The permeability of the generated hydrogel matrices, prepared by photo cross-linking under different crosslinking conditions $\left(0.5-2 \mathrm{~J} / \mathrm{cm}^{2}\right)$, was tested by measuring binding kinetics of streptavidin on PEI-B modified surfaces. As transducer, a four-channel silicon nitride $\left(\mathrm{Si}_{3} \mathrm{~N}_{4}\right)$ waveguide based Mach-Zehnder interferometric (MZI) sensing platform is used, operating at 850 $\mathrm{nm}$ in TM-mode (Figure 1A).

In order to test the protein loading capacity, three different modification cases are probed (Figure 1B): 1) PEI-B receptors incorporated in a hydrogel film, deposited by spin-coating, 2) $\mathrm{PEI}-\mathrm{B}$ receptors incorporated in a hydrogel film, deposited by inkjet-printing and 3) PEI-B receptors in a spin-coated hydrogel film combined with a PEI-B modified surface. The $\mathrm{PEI}-\mathrm{B}$ concentration in the hydrogel precursor solution is varied in the range of $0-0.4 \mathrm{mg} / \mathrm{mL}$.

For cases 1 and 2 the streptavidin binding signal increases in a linear fashion with the PEI$B$ concentration in the applied hydrogel precursor solution. Comparing the results for receptor loaded hydrogels prepared by spincoating and inkjet printing shows that the obtained sensor signal is independent of the deposition method (Figure 1B). The highest 
signal is achieved for case 3 , combining a PEIB-modified surface with a highly PEI-B loaded hydrogel matrix $(0.4 \mathrm{mg} / \mathrm{mL}$, case 3$)$.
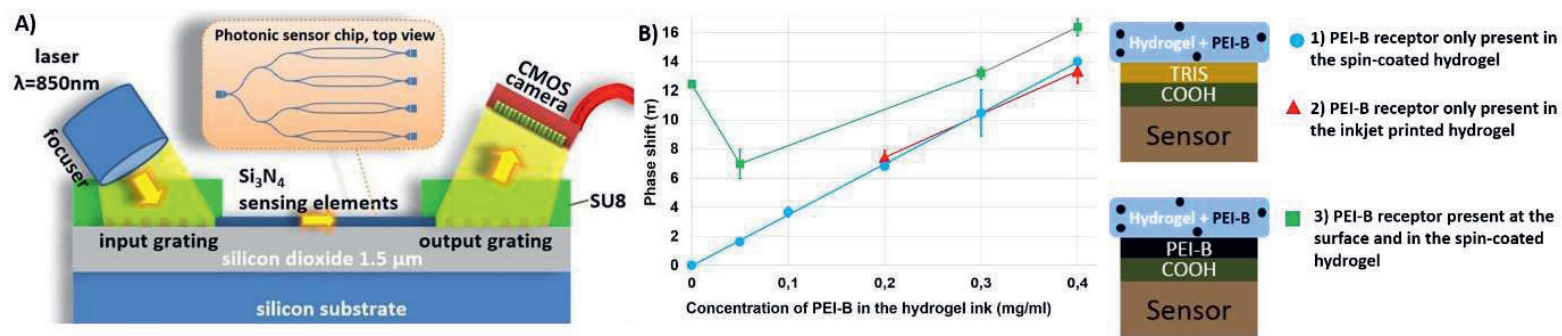

Fig.1. A) Schematics of the silicon nitride based, four-channel MZI photonic sensing platform; B) Results for streptavidin sensing, averaging four measurements for each point, and schematics of sensor modification.

\section{References}

[1] K. Tiefenthaler, W. Likosz, Journal of the Optical Society of America B 6, 209-220 (1989); doi: 10.1364/JOSAB.6.000209

[2] F. Strasser, E. Melnik, P. Muellner, P. JiménezMeneses, M. Nechvile, G. Koppitsch, P.

Lieberzeit, M. Laemmerhofer, R. Heer, R. Hainberger, Proceeding of SPIE 10231, 102312H (2017); doi: 10.1117/12.2267156 